\title{
Biosynthesis of trans-4-hydroxyproline by recombinant strains of Corynebacterium glutamicum and Escherichia coli
}

\author{
Yulan $\mathrm{Yi}^{1}$, Huakai Sheng ${ }^{1}$, Zhimin $\mathrm{Li}^{1,2^{*}}$ and Qin $\mathrm{Ye}^{1}$
}

\begin{abstract}
Background: Trans-4-hydroxy-L-proline (trans-Hyp), one of the hydroxyproline (Hyp) isomers, is a useful chiral building block in the production of many pharmaceuticals. Although there are some natural biosynthetic pathways of trans-Hyp existing in microorganisms, the yield is still too low to be scaled up for industrial applications. Until now the production of trans-Hyp is mainly from the acid hydrolysis of collagen. Due to the increasing environmental concerns on those severe chemical processes and complicated downstream separation, it is essential to explore some environment-friendly processes such as constructing new recombinant strains to develop efficient process for trans-Hyp production.

Result: In this study, the genes of trans-proline 4-hydroxylase (trans-P4H) from diverse resources were cloned and expressed in Corynebacterium glutamicum and Escherichia coli, respectively. The trans-Hyp production by these recombinant strains was investigated. The results showed that all the genes from different resources had been expressed actively. Both the recombinant C. glutamicum and E. coli strains could produce trans-Hyp in the absence of proline and 2-oxoglutarate.

Conclusions: The whole cell microbial systems for trans-Hyp production have been successfully constructed by introducing trans-P4H into C. glutamicum and E. coli. Although the highest yield was obtained in recombinant E. coli, using recombinant C. glutamicum strains to produce trans-Hyp was a new attempt.
\end{abstract}

Keywords: Trans-4-hydroxy-L-proline, Recombinant Corynebacterium glutamicum, Recombinant Escherichia coli, Proline 4-hydroxylases

\section{Background}

Hydroxyproline (Hyp) is a specific amino acid component of collagen. The amount of Hyps varies from 80 to 100 residues per 1000 residues in mammalian collagen, which can be used to estimate collagen content and act as an important indicator to collagen quality [1]. There are five naturally occurring Hyps, including three diastereomers of 4-hydroxyproline and two diastereomers of 3-hydroxyproline. Among them, trans-4-hydroxy-Lproline is the most abundant component in the constitution of collagen and can enhance the procollagen synthesis. Its derivative $\mathrm{N}$-acetyl trans-4-hydroxyproline (oxaceprol)

\footnotetext{
* Correspondence: lizm@ecust.edu.cn

'State Key Laboratory of Bioreactor Engineering, East China University of Science and Technology, 130 Meilong Road, Shanghai 200237, China

${ }^{2}$ Shanghai Collaborative Innovation Center for Biomanufacturing Technology, Shanghai, China
}

\section{Biomed Central

is an atypical inhibitor of inflammation and useful for the treatment of diseases affecting the connective tissues such as osteoarthritis [2]. Trans-Hyp has been widely used in medicine, biochemistry, food, cosmetic and other aspects of industry [3]. Additionally, trans-Hyp has also been found in the composition of some secondary metabolites such as actinomycins and echinocandins [4].

Trans-Hyp is manufactured industrially most by acid hydrolysis of mammalian collagen because of its rich amount in the collagen. However, it obviously results in many environmental issues and brings great difficulties into the down stream processing [5]. There are several identified pathways of hydroxyproline biosynthesis. In animal tissue, 4-hydroxyproline is catalyzed by prolyl 4-hydroxylase, which takes peptidyl proline as substrate rather than free proline [6]. 4-hydroxy-2-oxoglutaric acid can be enzymatically transformed to hydroxyproline [7]. 
Some bacteria or fungi have been found to form hydroxyproline via fermentation directly [8]. Although the titer of product is low, these findings show the possibility of utilizing biological processes to produce trans-Hyp.

The proline 4-hydroxylases ( $\mathrm{P} 4 \mathrm{Hs}$ ) have been identified from several microbial strains, which can catalyze the hydroxylation of L-proline at the 4-position to produce trans-Hyp in the presence of 2-oxoglutarate, oxygen and ferrous ion [9-11]. $\mathrm{P} 4 \mathrm{Hs}$ have an optimum $\mathrm{pH}$ range of 6.0 to 7.5 and temperature range of $30^{\circ} \mathrm{C}$ to $40^{\circ} \mathrm{C}$. Its activity is inhibited by metal ions such as $\mathrm{Zn}^{2+}$ and $\mathrm{Cu}^{2+}$. Lawrence et al. have studied the effect of co-substrates on the hydroxylation of L-proline by $\mathrm{P} 4 \mathrm{H}$ and pointed that 2-oxoglutarate was essential for proline hydroxylation since the replacement of 2-oxoglutarate with 2-oxopentanoate, 2-oxoadipate, pyruvate or 2-oxomalonate (all at $0.5 \mathrm{mM}$ ) led to no detectable hydroxylation of L-proline [10]. Although 2-oxoglutarate as the oxygen donator is required for hydroxylation of L-proline to 4-hydroxy-L-proline in vitro, it is unnecessary to add extra 2-oxoglutarate in vivo in the production of 4-hydroxy-L-proline by recombinant strains. 2-oxoglutarate is a key metabolic intermediate in the tricarboxylic acid cycle (TCA cycle) in Escherichia coli strain, which can result in the formation of hydroxyproline from glucose and proline directly [12]. Shibasaki et al. have analyzed the possible metabolic pathways of 2-oxoglutatate. They concluded that 2-oxoglutatate can be supplied either through the action of proline dehydrogenase (PutA) from L-proline or through the action of isocitric dehydrogenase (Icd) from glucose. The addition of L-proline to a glucose-containing minimal medium had a positive effect on both the proline 4-hydroxylase activity and production level [13]. But the availability of intracellular proline may still be limited because the biosynthesis of proline in wild type E. coli is strictly regulated to very low level. Thus, the precursor and co-factor in the microbial production of hydroxyproline need to be considered simultaneously.

Corynebacterium glutamicum is one of the most important industrial microorganisms and widely used in amino acids, vitamins and nucleic acids production [14]. Leuchtenberger et al. has summarized the commercial application of C. glutamicum to the fermentative production of amino acids [15]. Lee et al. has reported a novel glutamate and proline producing method through the utilization of phenol in C. glutamicum [16]. Masaaki Wachi reported a strategy for optimizing the industrial production of amino acids by reinforcing the export systems of C. glutamicum [17]. The metabolic pathways of amino acids are sophisticated and controlled tightly in C. glutamicum. But C. glutamicum as the platform of amino acid production has been studied in details and there are lots of molecular tools used for its genetic modifications, which contribute to C. glutamicum as one of the most popular host systems [18,19]. Additionally, Ikeda et al. [20] and Kalinowski et al. [21] have completed genome sequencing of C. glutamicum ATCC13032, which made C. glutamicum into a new era of system biology. To overproduce amino acids by $C$. glutamicum, not only the modification of biosynthetic pathway and regulation mechanism but also the transportation of amino acids plays significant roles in the final yield of a particular amino acid [22,23].

To produce trans-Hyp by microbial fermentation, it is essential to contain both the proline pathway and subsequent hydroxylation activity in the microbial cells. Considering the metabolic pathway in C. glutamicum or E. coli (Figure 1), proline and 2-oxoglutarate can be produced from glucose. NADPH and ATP can also be regenerated during the glucose metabolism. Although there are some efforts being conducted in E. coli, for example, Hyp was produced from glucose using a recombinant $E$. coli by introducing a proline 4-hydroxylase and the mutated $p r o B$ (encodingy-glutamyl kinase) gene encoding the feedback resistant enzyme [24]. However, the development of recombinant C. glutamicum for hydroxyproline secretion has never been reported. In this study, genes of trans- $\mathrm{P} 4 \mathrm{H}$ from diverse sources were screened and cloned into different C. glutamicum strains. The recombinant $E$. coli expressed different genes were also investigated. Meanwhile, a surprisingly high yield in shake flasks by recombinant $E$. coli without extra addition of L-proline was achieved.

\section{Results and discussion}

\section{Construction of trans-Hyp producing recombinant strains}

There are several genes being speculated as putative L-proline 4-hydroxylase gene in the database, including genes in Pseudomonas stutzeri, Janthinobacterium sp., Bordetella bronchiseptica RB50, Bradyrhizobium japonicum, Achromobacter xylosoxidans C54 and Dactylosporangium. sp. Using PCR, we cloned and obtained the putative genes of $\mathrm{P} 4 \mathrm{H}$ from $P$. stutzer and B. bronchiseptica $R B 50$, named $p 4 h P$ and $p 4 h B$. They were ligated to the corresponding plasmids after digestion and converted to C. glutamicum and E. coli, respectively. The length of $p 4 h P$ was 918 bps while $p 4 h B$ was 924 bps. These sequences were $100 \%$ identical to the reported genes in NCBI. The gene of trans-P4H from Dactylosporangium $s p$. $(p 4 h D)$ had been expressed in E. coli successfully and can transform L-proline with good enzymatic properties [11-13,24-26]. The length of $p 4 h D$ was 816 bps encoding a 272-amino-acid polypeptide with the molecular weight of 29,715 daltons $[11,26]$. In this study, $p 4 h D$ was applied with some modifications on the nuclear bases. The original gene sequence of $p 4 h D$ was analyzed (http:// www.kazusa.or.jp/codon/) and the results showed there were some rare codons for both C. glutamicum and 


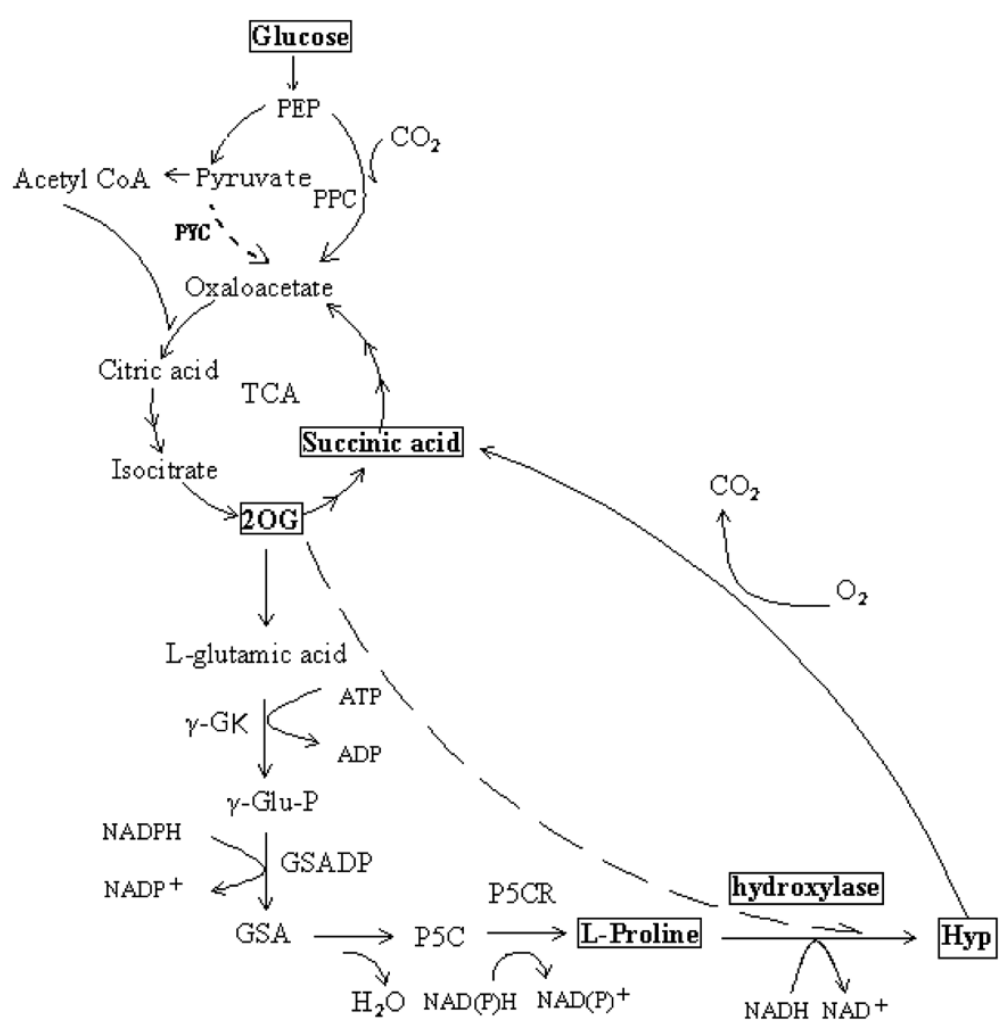

Figure 1 The metabolism of trans-4-hydroxyproline in recombinant bacteria. 2OG: 2 - 2-Ketoglutaric acid; $\gamma$-GK: $\gamma$ - Glutamyl kinase; $\gamma$-Glu-P. Y-Glutamyl phosphate; GSADH; Glutamyl phosphate reductase; GSA: Glutamyl semialdehyde; P5C: Pyrroline - 5 - carboxylic acid; P5CR: P5C reductase; Hyp: Hydroxyproline.

E. coli. It has been reported that rare codons are strongly associated with low level of protein expression [27]. Codon optimization for heterologous protein expression has often been shown to drastically increase protein expression [28]. Thus, the rare codons of $p 4 h D$ gene were substituted for those used with high frequency in C. glutamicum and the $\mathrm{GC}$ content was adjusted from $73 \%$ to $61 \%$ through synonymous conversion, which was close to that of $C$ glutamicum. The modified gene of $p 4 h D$ was synthesized according to the above modifications (Additional file 1).

The expression of $\mathrm{P} 4 \mathrm{H}$ is one of the important aspects on the construction of trans-Hyp biosynthetic pathway. Figure 2 shows the SDS-PAGE of trans-P4Hs expressed in recombinant $C$. glutamicum and E. coli. All the recombinant trans-P4Hs were expressed as soluble proteins without inclusion bodies. It was obviously that the recombinant trans-P4Hs in E. coli were expressed much more than those in C. glutamicum (Figure 2). Many factors have influences on the expression of foreign proteins including promoters, the host-vector system and cultural conditions etc. $[29,30]$. Since it was the first to express trans-P4Hs in C. glutamicum, more comprehensive studies such as promoter selection and culture condition optimization will be considered in our future work.

\section{Comparison of $\mathrm{P} 4 \mathrm{H}$ activities}

Oxygenases are widely applied in industry since they can catalyze the highly specific oxyfunctionalization of unactivated $\mathrm{C}-\mathrm{H}$ bonds under mild conditions, especially transferring molecular oxygen to a substrate [31]. P4H belongs to a family of 2-oxoacid-dependent dioxygenase, which is a monomeric protein and utilizes the monomeric rather than polymeric substrates [10]. The activities of transP4Hs using recombinant whole cells in this study were measured (Table 1). Our data indicated that the expressed protein level and enzymatic activity was higher at $30^{\circ} \mathrm{C}$. The results also showed that the plasmids were very stable as the plasmid stabilities of recombinant E. coli and $C$. glutamicum strains were all more than $98 \%$ at the end of fermentation.

The recombinant cells with expressing of different genes showed different levels of catalytic activities toward L-proline. The activity of trans-P4H expressed by E. coli $\mathrm{BL} 21 / \mathrm{pET} 28 \mathrm{a}-p 4 h D$ was the highest among all the constructed recombinant strains. The new cloned and expressed genes from P. stutzeri and B. bronchiseptica also showed interested activities. As for different host strains, E. coli represented better than C. glutamicum, which may be related to the performance of corresponding 


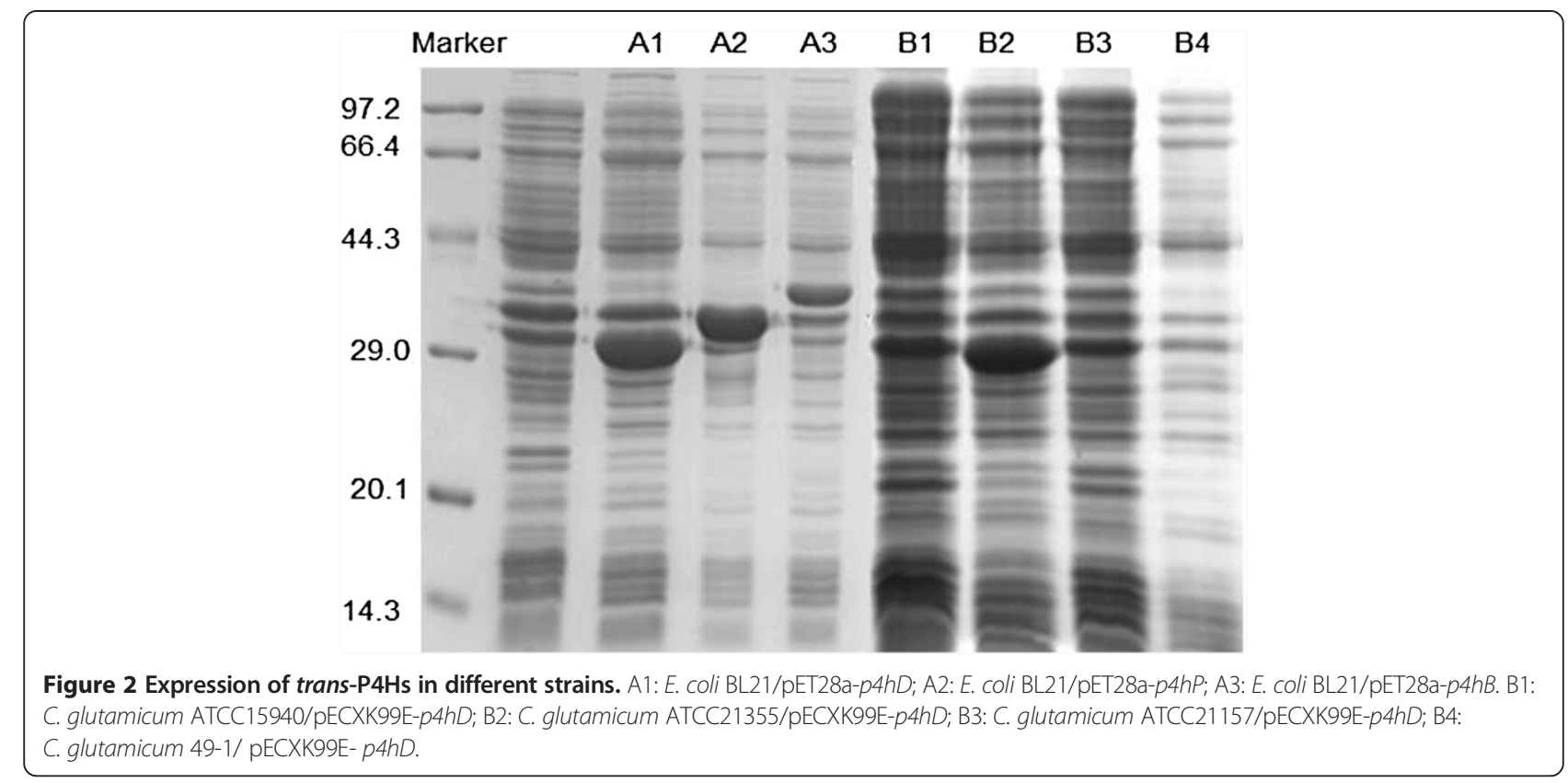

plasmid. Four L-proline producing strains of C. glutamicum were used as expression host strains and the resulted recombinant strains showed different enzymatic activities. The highest specific enzymatic activity among C. glutamicum strains was $40.7 \mathrm{U} / \mathrm{mg} \cdot$ wet cell by $C$. glutamicum ATCC13032/pEC-XK99E-p4hB. However, the specific enzymatic activity of recombinant $E$. coli/pET28a $-p 4 h D$ was up to $60.4 \mathrm{U} / \mathrm{mg}$ - wet cell. The growth of three recombinant $E$. coli strains was similar. But there was significant difference among the recombinant $C$. glutamicum strains. The recombinant $C$. glutamicum strains with higher specific enzymatic activities grew less than those with lower specific enzymatic activities. Additionally, the enzymatic activity of E. coli BL21/pET28a - p4hD was similar to that of $E$. coli W1485/pWFH1 and higher than that of $E$. coli BL21/pET24-p4h1 of [12,13]. The $p 4 h D$ in E. coli W1485/ pWFH1 was the original one in Dactylosporangium sp., while $p 4 h D$ in $E$. coli BL21/pET24-p4h1 was modified. Although the codon optimization in this study was designed for C. glutamicum, the results indicated that it was also successfully in $E$. coli.

\section{Trans-Hyp production in flasks}

The production of trans-Hyp by different recombinant C. glutamicum and E. coli strains was also shown in Table 1 . The yields of trans-Hyp by these recombinant strains depended both on the enzymatic activity of $\mathrm{P} 4 \mathrm{H}$ and cell growth. E. coli BL21/ pET28a-p4hD had the

Table 1 Comparison of trans-P4Hs activities and trans-Hyp production by different recombinant C. glutamicum and E. coli strains

\begin{tabular}{lccc}
\hline Strains & Specific activities (U/mg $\cdot$ wet cell weight) & Trans-Hyp (g/L) & OD600 (OD620)* \\
\hline C. glutamicum ATCC13032/pEC-XK99E- p4hD & $37.4 \pm 1.4$ & $0.072 \pm 0.001$ & $5.5 \pm 0.7$ \\
C. glutamicum ATCC13032/pEC-XK99E- p4hP & $20.7 \pm 1.1$ & $0.106 \pm 0.002$ & $7.3 \pm 0.5$ \\
C. glutamicum ATCC13032/pEC-XK99E-p4hB & $40.7 \pm 0.8$ & $0.079 \pm 0.016$ & $5.4 \pm 0.03$ \\
C. glutamicum ATCC15940/pEC-XK99E- p4hD & $12.9 \pm 0.5$ & $0.103 \pm 0.001$ & $14.0 \pm 0.2$ \\
C. glutamicum ATCC21355/pEC-XK99E- p4hD & $35.9 \pm 0.1$ & $0.087 \pm 0.005$ & $6.6 \pm 0.2$ \\
C. glutamicum ATCC21157/pEC-XK99E- p4hD & $12.3 \pm 0.9$ & $0.112 \pm 0.004$ & $13.3 \pm 0.1$ \\
C. glutamicum 49-1/pEC-XK99E-p4hD & $12.4 \pm 0.6$ & $0.113 \pm 0.001$ & $13.8 \pm 0.5$ \\
E. coli BL21/pET-28a -p4hD & $60.4 \pm 1.8$ & $0.470 \pm 0.028$ & $6.5 \pm 0.2$ \\
E. coli BL21/pET-28a -p4hP & $22.2 \pm 0.5$ & $0.126 \pm 0.007$ & $7.3 \pm 0.05$ \\
E. coli BL21/pET-28a -p4hB & $50.0 \pm 2.2$ & $0.115 \pm 0.006$ & $6.9 \pm 0.1$ \\
\hline
\end{tabular}

*Optical density at $600 \mathrm{~nm}$ for E. coli and $620 \mathrm{~nm}$ for C. glutamicum. 
highest yield, which was coincided of its specific enzymatic activity. Although the recombinant E. coli strains grew similarly in the production medium, there was significant difference in the production of trans-Hyp which did not keep the same level with the specific enzymatic activities. The productions of trans-Hyp by recombinant C. glutamicum strains were also much less than that of E. coli BL21/pET28a-p4hD. It was due to both the less expression of trans-P4H and less cell growth in C. glutamicum. The L-proline production of four C. glutamicum strains was also less than $1 \mathrm{~g} / \mathrm{L}$. There was little difference of trans-Hyp production among the recombinant strains of C. glutamicum with same gene $p 4 h D$, despite that some strains had better enzymatic performance and proline production.

Using the recombinant strains to directly synthesize trans-Hyp from glucose via fermentation was achieved since both the enzymes and precursors needed in the process were available. The over expressed foreign transP4Hs catalyzed the hydroxylation of L-proline at the trans-4 position, while $2-$ ketoglutarate was supplied by glucose through TCA cycle and then oxidatively decarboxylated to succinate (Figure 1). It was reported that proline was demanded in the production of Hyp by recombinant $E$. coli only with $p 4 h$ gene. The carbon in proline added during the fermentation only flowed into amino acids synthesized from TCA cycle intermediates and not into gluconeogenesis [13]. However, the accumulated Hyp was at a relative high level even without the addition of proline in this study. It could be understood that Corynebacterium had the powerful biosynthetic pathway of proline [32,33]. The biosynthetic pathway of proline has also been identified in E. coli, which may contribute to the synthesis of trans-Hyp by recombinant $E$. coli strains. The modification of proline pathway in $E$. coli enhanced the yield of Hyp, whereas the formation of Hyp can also relieve the feedback inhibition of proline [24]. The amount of proline (0-4 $\mathrm{mM}$ ) promoted the production of transHyp. However, continuously addition of L-proline didn't improve the production yield significantly (Table 2). In this study, the time of cultivation was significantly less than those reported in the literatures, which might attribute to the different media used and also indicated there was great potential with optimization. In fact, $2.28 \mathrm{~g} / \mathrm{L}$ of trans-Hyp was produced by recombinant $E$. coli without adding L-proline in flasks with a little modification of media and $6.72 \mathrm{~g} / \mathrm{L}$ was achieved by supplement only $4 \mathrm{mM}$ L-proline.

Table 2 Hyp production under different L-proline supplementation

\begin{tabular}{ccccccc}
\hline $\begin{array}{c}\text { Supplementary addition } \\
\text { of L- proline }(\mathbf{m M})\end{array}$ & $\mathbf{0}$ & $\mathbf{1}$ & $\mathbf{2}$ & $\mathbf{4}$ & $\mathbf{8}$ & $\mathbf{1 2}$ \\
\hline Hyp $(\mathrm{g} / \mathrm{L})$ & 2.28 & 2.81 & 3.25 & 6.72 & 5.56 & 6.32 \\
\hline
\end{tabular}

In order to further increase the biosynthesis of transHyp by recombinant $C$. glutamicum and E. coli, alternative approaches should be considered as well. In E. coli, the degradation of proline should be overcome. Although the trans-Hyp production by a putA mutant of $E$. coli was not improved furthermore, the yield based on the proline utilized was enhanced greatly. In both C. glutamicum and $E$. coli, the expression of recombinant $\mathrm{P} 4 \mathrm{H}$ as one of the oxygenases is involved in the physiological metabolism of host cells including the cofactor, co-substrate and oxygen. Moreover, without a powerful proline synthetic pathway in $E$. coli, the availability and transportation of substrate will limit the transformation seriously.

\section{Conclusions}

In this study, two new and a modified trans-P4Hs were expressed in C. glutamicum and E. coli successfully. Different amount of trans-Hyp were produced by these recombinant strains detected. Although the yield in recombinant C. glutamicum was less than that in recombinant E. coli, C. glutamicum as a native proline producing strain was worthy of further optimizing. This is the first report of producing trans-Hyp by introducing L-proline 4-hydroxylase into C. glutamicum.

\section{Methods}

\section{Strains and plasmids}

The bacterial strains and plasmids used in this study are listed in Table 3. E. coli BL21(DE3) and five C. glutamicum strains among which four were reported to produce L-proline were used as hosts. Plasmids pET-28a and pEC-XK99E were applied as the vectors respectively. Trans- 4-hydroxy-L-proline was purchased from SigmaAldrich Trading Co., Ltd.

\section{Construction of recombinant strains}

The full sequences of $p 4 h$ encoding predicted hydroxylases were amplified from the strains' genomic DNA as followed in Table 4. The primers used in this study for gene cloning and plasmid construction were also as listed in Table 4, which were incorporated SalI/EcoRI restriction sites for recombinant $E$. coli and SalI/XbaI for recombinant C. glutamicum. The PCR products were digested with restriction enzymes above and inserted into the vector pET-28a and pEC-XK99E respectively, which resulted into the expression plasmid pET-28a-p4h and pEC-XK99E- $p 4 h$. The inserted fragment was sequenced and verified the identity to the anticipated sequence. Recombinant plasmid was transferred into $E$. coli competent cells by chemical $\mathrm{CaCl}_{2}$ method. The preparation of C. glutamicum competent cell and electro transformation of exogenous gene were conducted according to the method in reference [34]. 
Table 3 Strains and plasmids used in this study

\begin{tabular}{|c|c|c|}
\hline Strains \& plasmids & Properties & Source/reference \\
\hline \multicolumn{3}{|l|}{ E. coli } \\
\hline JM109 & Cloning host & Our laboratory \\
\hline BL21(DE3) & $F-$, ompt, hsdS(rBB-mB-), gal, dcm (DE3) & Our laboratory \\
\hline \multicolumn{3}{|l|}{ C. glutamicum } \\
\hline ATCC13032 & Wild-type & Our laboratory \\
\hline ATCC 15940 & L-Proline production & Our laboratory \\
\hline ATCC 21355 & L-Proline production & [27] \\
\hline ATCC 21157 & L-Proline production & [27] \\
\hline 49-1 Plasmids & L-Proline production & Our laboratory \\
\hline pET-28a & $\mathrm{His}_{4}$-tag, T7 promoter, Kan ${ }^{\mathrm{r}}$ & Our laboratory \\
\hline pEC-XK99E & E. coli - C. glutamicum shuttle expression vector, Kan ${ }^{r}$ & Our laboratory \\
\hline pET-28a-p4hP & pET-28a containing the $p 4 h$ gene from $P$. stutzeri & This study \\
\hline pEC-XK99E-p4hP & pEC-XK99E containing the $p 4 h$ gene from $P$. stutzeri & This study \\
\hline pET-28a-p4hB & pET-28a containing the $p 4 h$ gene from $B$. bronchiseptica & This study \\
\hline pEC-XK99E-p4hB & pEC-XK99E containing the $p 4 h$ gene from $B$. bronchiseptica & This study \\
\hline pET-28a- p4hD & pET-28a containing the $p 4 h$ gene from Dactylosporangium sp. & This study \\
\hline pEC-XK99E-p4hD & pEC-XK99E containing the $p 4 h$ gene from Dactylosporangium sp. & This study \\
\hline
\end{tabular}

$r$ indicates resistant.

\section{Media}

Luria broth (LB) medium, tryptone $10 \mathrm{~g} / \mathrm{L}$; yeast extract $5 \mathrm{~g} / \mathrm{L}$; solium chloride $10 \mathrm{~g} / \mathrm{L}$, was used for seed cultivation of $E$. coli strains. LBG medium containing $1 \%$ glucose additionally was used for C. glutamicum seed cultivation.

The medium (MEC) for batch culture of $E$. coli in shake flasks contained: glucose $10 \mathrm{~g} / \mathrm{L}$, glycerol $5 \mathrm{~g} / \mathrm{L}, \mathrm{CO}\left(\mathrm{NH}_{2}\right)_{2}$ $10 \mathrm{~g} / \mathrm{L}$, yeast extract $10 \mathrm{~g} / \mathrm{L}, \mathrm{K}_{2} \mathrm{HPO}_{4} 1 \mathrm{~g} / \mathrm{L}, \mathrm{NaCl} 2 \mathrm{~g} / \mathrm{L}$, $\mathrm{MgSO}_{4} \cdot 7 \mathrm{H}_{2} \mathrm{O} 0.2 \mathrm{~g} / \mathrm{L}, \mathrm{FeSO}_{4} \cdot 7 \mathrm{H}_{2} \mathrm{O} 1 \mathrm{mM}, \mathrm{MnSO}_{4}$. $4 \mathrm{H}_{2} \mathrm{O} 10 \mathrm{mg} / \mathrm{L}, \mathrm{ZnSO}_{4} \cdot 7 \mathrm{H}_{2} \mathrm{O} 10 \mathrm{mg} / \mathrm{L}, \mathrm{VB}_{1} 200 \mathrm{ug} / \mathrm{L}$.

The medium (MCG) for batch culture of C. glutamicum in shake flasks contained: glucose $10 \mathrm{~g} / \mathrm{L}$; glycerol $5 \mathrm{~g} / \mathrm{L}$;
$\mathrm{CO}\left(\mathrm{NH}_{2}\right)_{2} 10 \mathrm{~g} / \mathrm{L}$; corn syrup $15 \mathrm{~g} / \mathrm{L} ; \mathrm{K}_{2} \mathrm{HPO}_{4} 1 \mathrm{~g} / \mathrm{L} ; \mathrm{NaCl}$ $2 \mathrm{~g} / \mathrm{L} ; \mathrm{MgSO}_{4} \cdot 7 \mathrm{H}_{2} \mathrm{O} 0.2 \mathrm{~g} / \mathrm{L} ; \mathrm{FeSO}_{4} \cdot 7 \mathrm{H}_{2} \mathrm{O} 1 \mathrm{mM}$; $\mathrm{MnSO}_{4} \cdot 4 \mathrm{H}_{2} \mathrm{O} 10 \mathrm{mg} / \mathrm{L} ; \mathrm{ZnSO}_{4} \cdot 7 \mathrm{H}_{2} \mathrm{O} 10 \mathrm{mg} / \mathrm{L} ; \mathrm{VB}_{1}$ $200 \mathrm{mg} / \mathrm{L}$; ethyl alcohol absolute $1.5 \%$.

\section{Cultivations}

The seed culture of $E$. coli strains was prepared by transferring $1 \mathrm{ml}$ of glycerol stock to $30 \mathrm{ml}$ of LB medium in a 250 -ml flask, which was incubated overnight at $37^{\circ} \mathrm{C}$ and $220 \mathrm{rpm}$. Then $6 \%$ of seed culture was inoculated into $30 \mathrm{ml}$ of MEC medium in a $250 \mathrm{ml}$ flask and incubated at $37^{\circ} \mathrm{C}$ and $220 \mathrm{rpm}$ for about $36 \mathrm{~h}$. The initial $\mathrm{pH}$ of the

Table 4 Primers used in this study for gene cloning and plasmid construction

\begin{tabular}{|c|c|c|}
\hline Primer name & Sequences $\left(5^{\prime} \rightarrow 3^{\prime}\right)$ & Source \\
\hline p4hP-pET-28a-S & TGTAGAATTCATGATCTCACCTGCGCA & P. stutzeri \\
\hline p4hP-pET-28a-A & ATATAAGCTTCTAGCTGCCGACCAGCTTC & \\
\hline p4hP -pEC-XK99E-S & TAATGAATTCGTGAACCCTATGCAAGC & P. stutzeri \\
\hline p4hP -pEC-XK99E -A & ATAGTCTAGATCAGAGATACTGTTGCGG & \\
\hline p4hB-pET-28a-S & TGATGAATTCCTAGCGCTCGACCAGTTT & B. bronchiseptica \\
\hline$p 4 h B-p E T-28 a-A$ & CTGCAAGCTTATGATTTCACCTGCTCAGG & \\
\hline p4hB -pEC-XK99E-S & TATAGAATTCATGATCTCACCTGCCCAG & B. bronchiseptica \\
\hline p4hB -pEC-XK99E -A & TGTCTCTAGATTATTCCACCAGCTTCAG & \\
\hline p4hD -pET-28a-S & TATAGAGCTCATGCTGACTCCGACCGA & Dactylosporangium sp. \\
\hline p4hD -pET-28a-A & GATCAAGCTITTAAACTGGCTGGGCAAG & \\
\hline p4hD -pEC-XK99E-S & TATAGAGCTCATGCTGACTCCGACCGA & Dactylosporangium sp. \\
\hline p4hD -pEC-XK99E-A & GATCTCTAGATTAAACTGGCTGGGCAAG & \\
\hline
\end{tabular}


medium was adjusted to 7.4. Induction (0.5 mM IPTG) was performed when the optical density was around 0.5 , and the growth temperature was reduced from initial $37^{\circ} \mathrm{C}$ to $30^{\circ} \mathrm{C}$. The cultivation of C. glutamicum was similar to that of $E$. coli expect that they were conducted at $30^{\circ} \mathrm{C}$ in the whole process using MCG medium. Experiments were performed in parallel on the same media without any induction.

\section{Trans- $\mathrm{P} 4 \mathrm{H}$ activities}

The intracellelular trans- $\mathrm{P} 4 \mathrm{H}$ activities were measured by the whole-cell reaction procedures. After 8 hours induction in fermentation medium, cells were harvested by centrifugation at $12000 \times \mathrm{g}$ for $20 \mathrm{~min}$. The harvested cells were resuspended in the reaction mixture as followed. Each reaction mixture contained $80 \mathrm{mM}$ 2-[N- morpholino] ethanesulfonic acid (MES) buffer (pH 6.5), 4 mM L-proline, 8 mM 2 - ketoglutarate, $2 \mathrm{mM}$ FeSO4, $4 \mathrm{mM}$ L-ascorbic acid The final cell concentration was about $100 \mathrm{~g}$ wet weight/L. The reaction mixtures were incubated at $35^{\circ} \mathrm{C}$ for $10 \mathrm{~min}$ and then cellular activity was inactivated completely by heat treatment at $100^{\circ} \mathrm{C}$ and 5 minutes. The amount of trans-4-hydroxy-Lproline in the supernatant of each mixture after centrifugation was determined. The amount of the enzyme which forms $1 \mathrm{nmol}$ of Hyp in one minute was defined as $1 \mathrm{U}$.

\section{Analytical methods}

The cell concentration was determined by measuring the optical density of appropriately diluted sample at $600 \mathrm{~nm}$ (E. coli, $\left.\mathrm{OD}_{600}\right)$ and $620 \mathrm{~nm}\left(C\right.$. glutamicum $\left.\mathrm{OD}_{620}\right)$ with a UV-visible spectroscopy system (Xinmao, Shanghai, China). Hyp was oxidized by Chloramine $\mathrm{T}$ and analyzed using spectrophotometric determination [35].

\section{Statistical analysis}

All measurements for growth, trans-Hyp production and trans- $\mathrm{P} 4 \mathrm{H}$ activity were performed in triplicate, and the data were averaged and presented as the mean \pm standard deviation.

\section{Additional file}

\section{Additional file 1: Supplement.}

\section{Competing interests}

The authors declare that they have no competing interests.

\section{Authors' contributions}

YY participated in the design and conducted the experiments in the study, and drafted the manuscript. HS participated in the analysis of proline and hydroxyproline. ZL conceived the study and helped to draft the manuscript. QY discussed the experiments and manuscript. All authors read and approved the final manuscript.

\section{Acknowledgements}

This study was supported by the National High Technology Research and Development Program of China (Grant No's. 2012AA022104 \& 2012AA021205), the Fundamental Research Funds for the Central Universities (Grant No's.
222201313007 \& 222201314048), the National Special Fund for State Key Laboratory of Bioreactor Engineering (No.2060204).

Received: 7 February 2014 Accepted: 15 May 2014

Published: 19 May 2014

\section{References}

1. Kuttan R, Radhakrishnan AN: Biochemistry of the hydroxyprolines. Adv Enzymol 1973, 37:273-347.

2. Mai Hoa BT, Hibi T, Nasuno R, Matsuo G, Sasano Y, Takagi H: Production of $\mathrm{N}$-acetyl cis-4-hydroxy-L-proline by the yeast $\mathrm{N}$-acetyltransferase Mpr1. J Biosci Bioeng 2012, 114(2):160-165.

3. Remuzon P: Trans 4-Hydroxy-L-proline, a novel and versatile chiral starting block. Tetrahedron 1996, 52:13803-13835.

4. Wichmann CF, Liesch JM, Schwartz RE: L-671,329, a new antifungal agent. II. Structure determination. Antibiot 1989, 42(2):168-173.

5. Izumi Y, Chibata I, Itah T: Herstellung und Verwendung von Aminosäuren. Angew Chem 1978, 90(3):187-194.

6. Gorres KL, Raines RT: Prolyl 4-hydroxylase. Crit Rev Biochem Mol Biol 2010, 45:106-124.

7. Hashimoto $S$, Katsumata R, Ochiai K: Process for producing optically active 4-hydroxy-2-ketoglutaric acid using microorganisms. US Patent. 5,607,848 [P]. 1997-3-4.

8. Serizawa N, Matsuoka T. Hosoya T, Furuya K: Fermentation production of trans-4-hydroxy-L-proline by Clonostachys cylindrospora. Biosci Biotech Biochem 1995, 59(3):555-557.

9. Onishi M, Okumura R, Okamoto R, Ishikura T: Proline hydroxylation by cell free extract of a streptomycete. Biochem Biophys Res Commun 1984 . 120(1):45-51.

10. Lawrence CC, Sobey WJ, Field RA, Baldwin JE, Schofield CJ: Purification and initial characterization of proline 4-hydroxylase from Streptomyces griseoviridus P8648: a 2-oxoacid, ferrous-dependent dioxygenase involved in etamycin biosynthesis. Biochem J 1996, 313(1):185-191.

11. Baldwin JE, Field RA, Lawrence CC, Lee V, Robinson JK, Schofield CJ: Substrate specificity of proline 4-hydroxylase: chemical and enzymatic synthesis of $2 S, 3 R, 4 S$-epoxyproline. Tetrahedron Lett 1994 35(26):4649-4652.

12. Shibasaki T, Mori H, Ozaki A: Enzymatic production of trans-4-Hydroxy -Lproline by region- and stereospecific hydroxylation of L-proline. Bios $\mathrm{C} i$ Biotechno Biochem 2000, 64(4):746-750.

13. Falcioni F, Blamk LM, Oliver F, Andreas K, Bruno B, Schmid A: Proline availability regulates proline-4-hydroxylase synthesis and substrate uptake in proline-hydroxylating recombinant Escherichia coli. Appl Environ Microbiol 2013, 79:3091-3100

14. Eggeling $L$, Bott M: Handbook of Corynebacterium glutamicum. In Boca Raton: CRC Press: 2005:1-616

15. Wolfgang L, Klaus H, Karlheinz D: Biotechnological production of amino acids and derivatives: current status and prospects. Appl Microbiol Biotechnol 2005, 69:1-8.

16. Soo YL, Yang HK, Jiho M: Conversion of phenol to glutamate and proline in Corynebacterium glutamicum is regulated by transcriptional regulator ArgR. Appl Microbiol Biotechnol 2010, 85:713-720.

17. Masaaki W: Amino Acid Exporters in Corynebacterium glutamicum Microbiol Monographs 2013, 23:335-349.

18. Jiang LY, Chen SG, Zhang YY, Liu JZ: Metabolic evolution of Coryne- bacterium glutamicum for increased production of L-ornithine. $B M C$ Biotechnol 2013, 13:47.

19. Meiswinkela TM, Rittmannb D, Lindnera SN, Wendischa VF: Crude glycerolbased production of amino acids and putrescine by Corynebacterium glutamicum. Bioresour Technol 2013, 145:254-258.

20. Ikeda M, Nakagawa SL: The Corynebacterium glutamicum genome: features and impacts on biotechnological processes. Appl Microbiol Biotechnol 2003, 62(2/3) 99-109.

21. Kalinowski J, Bathe B, Bartels D, Bischoff N, Bott M, Burkovski A, Dusch N, Eggeling L, Eikmanns BJ, Gaigalat L, Goesmann A, Hartmann M, Huthmacher K, Kramer R, Linke B, McHardy AC, Meyer F, Mockel B, Pfefferle W, Puhler A, Rey DA, Ruchert C, Rupp O, Sahm H, Wendisch VF, Wiegrabe I, Tauch A: The complete Corynebacterium glutamicum ATCC 13032 genome sequence and its impact on the production of $\mathrm{L}$-aspartate-derived amino acids and vitamins. J Biotechnol 2003, 104(1/3):5-25. 
22. Becker J, Wittmann C: Systems and synthetic metabolic engineering for amino acid production-the heartbeat of industrial strain development. Curr Opin Biotechnol 2012, 23(5):718-726.

23. Ikeda M, Katsumata R: Transport of aromatic amino acids and its influence on overproduction of the amino acids in Corynebacterium glutamicum. J Ferment Bioeng 1994, 78(6):420-425.

24. Shibasaki T, Hashimoto S, Mori H, Ozaki A: Construction of a novel hydroxyproline-producing recombinant Escherichia coli by introducing a proline 4-hydroxylase gene. J Biosci Bioeng 2000, 90(5):522-525.

25. Shibasaki T, Mori H, Chiba S, Ozaki A: Microbial Proline 4-Hydroxylase Screening and Gene Cloning. Appl Environ Microbiol 1999, 65(9):4028-4031.

26. Klein C, Hüttel W: A simple procedure for selective hydroxylation of I-Proline and I-Pipecolic acid with recombinantly expressed proline hydroxylases. Adv Synth Catal 2011, 353(8):1375-1383.

27. Hayes CS, Bose B, Sauer RT: Stop codons preceded by rare arginine codons are efficient determinants of ssrA tagging in Escherichia coli. Proc Natl Acad Sci U S A 2002, 99(6):3440-3445.

28. Gustafsson C, Govindarajan S, Minshull J: Codon bias and heterologous protein expression. Trends Biotechnol 2004, 22:346-353.

29. Ahn SJ, Seo JS, Kim MS, Jeon SJ, Kim NY, Jang JH, Kim KH, Hong YK, Chung JK, Lee HH: Cloning, site-directed mutagenesis and expression of cathepsin L-like cysteine protease from Uronema marinum (Ciliophora: Scuticociliatida). Mol Biochem Parasitol 2007, 156(2):191-198.

30. Retnoningrum DS, Pramesti HT, Santika PY, Valerius O, Asjarie S, Suciati T: Codon optimization for high level expression of human bone morphogenetic protein- 2 in Escherichia coli. Protein Expr Purif 2012, 84:188-194

31. Blank LM, Ebert BE, Buehler K, Bühler B: Redox Biocatalysis and Metabolism: Molecular Mechanisms and Metabolic Network Analysis. Antioxid Redox 527 Signal 2010, 13:349-394.

32. Tsuchida T, Kubota K, Yoshinage F: Improvement of L-proline production by sulfaguanidine resistant mutants derived from L-glutamic acid-producing bacteria. Agric Biol Chem 1986, 50(9):2201-2207.

33. Nakanishi T, Hagino H: Process for producing L-proline by fermentation. US Patent 1984, 4:444,885.

34. Yu BQ, Shen W, Zhuge J: An improved method for integrative electro transformation of Corynebacterium glutamicum with xenogeneic DNA. China Biotechnol 2005, 25(2):78-81.

35. Yin MW, Nan YM, Wang XM: Improvement of the spectrophotometric method for the determination of hydroxyproline. J Henan Med Univ 1994, 29:74-77.

doi:10.1186/1472-6750-14-44

Cite this article as: Yi et al:: Biosynthesis of trans-4-hydroxyproline by recombinant strains of Corynebacterium glutamicum and Escherichia coli. BMC Biotechnology 2014 14:44.

\section{Submit your next manuscript to BioMed Central and take full advantage of:}

- Convenient online submission

- Thorough peer review

- No space constraints or color figure charges

- Immediate publication on acceptance

- Inclusion in PubMed, CAS, Scopus and Google Scholar

- Research which is freely available for redistribution 